\title{
An $f(R)$ Gravitation for Galactic Environments
}

\author{
Y. Sobouti \\ Institute for Advanced Studies in Basic Sciences, Zanjan, P. O. Box 45195-1159, Iran \\ sobouti@iasbs.ac.ir
}

We propose [Sobouti, arXiv:astro-ph/0603302] an $f(R)$ modification of GR, which admits of a modified Schwarzschild metric. In the weak field limit this amounts to adding a small logarithmic correction to the newtonian potential. A test star moving in such a spacetime acquires a constant speed at large distances. From observations of the velocity curves of spirals, this speed turns out to be proportional to the fourth root of the mass of the central body, in compliance with the Tully-Fisher relation. A variance of MOND emerges as a consequence of the formalism. The procedure is as follows: Extremizing the action $S=\frac{1}{2} \int f(R) \sqrt{-g} d^{4} x$ with respect to infinitesimal variations of $g_{\mu \nu}$ gives

$$
R_{\mu \nu}-\frac{1}{2} g_{\mu \nu} f / h=\left(h_{; \mu \nu}-h_{; \lambda}^{\lambda} g_{\mu \nu}\right) / h, \text { where } h=d f(R) / d R .
$$

For galactic purposes we addopt a spherically symmetric static metric

$$
d s^{2}=-B(r) d t^{2}+A(r) d r^{2}+r^{2}\left(d \theta^{2}+\sin ^{2} \theta d \varphi^{2}\right) .
$$

We are interested in those solutions of Eqs (0.1) and (0.2) which differ from those of the classical GR by small amounts. For GR one has $f=R$ and $h=1$. For our case we propose $h(r)=(r / s)^{\alpha} \approx 1+\alpha \ln (r / s)$, where $s$ is a length scale to be discussed shortly. Correct to the first order of smallness in $\alpha$, we find

$$
\begin{aligned}
& A^{-1}=(1-\alpha)^{-1}\left[1-(s / r)^{(1-\alpha / 2)}\right], \quad B=(r / s)^{\alpha} A^{-1}, \\
& R=3 \alpha r^{-2}, \quad f=R^{(1-\alpha / 2)} .
\end{aligned}
$$

For $\alpha=0, \mathrm{Eq}(0.3)$ is recognized as the Schwarzschild metric. Therefore, $s$ should be identified with the Schwarzschild radius, $2 G M / c^{2}$ of the body residing at the center. In the weak field limit, $A \approx 1$ and $B \approx 1+2 \phi(r) / c^{2}$, we find $\phi(r)=\frac{1}{2} \alpha c^{2} \ln r-G M / r$. A test star moving in such a potential experiences the acceleration $g=\frac{1}{2} \alpha c^{2} / r+G M / r^{2}$ towards the center and will have the circular velocity $v^{2}=\frac{1}{2} \alpha c^{2}+G M / r$. We have examined 42 spirals from a list of Sanders and Mc Gough [arXiv:astro-ph/0204521] for which asymptotic speeds, masses and sizes are available and have found $\alpha=2.8 \times$ $10^{-12}\left(M / M_{\odot}\right)^{1 / 2}$.

Concluding remarks: We have developed a spherically symmetric static $f(R)$ gravitation. In the weak field limit, a test star experiences a gravitational force $g=\sqrt{a_{0} g_{N}}+$ $g_{N}$, where $g_{N}=G M / r^{2}, a_{0}=\left[1.4 \times 10^{-12} c^{2}\right]^{2} / G M_{\odot}=1.2 \times 10^{-8} \mathrm{~cm} / \mathrm{sec}^{2}$. The main features of MOND are shared by this gravitational acceleration. The Tully-Fisher relation is also a consequence of the formalism.

Since the appearance of an earlier version of this work in arXiv, Mendosa and RosasGuevara [arXiv:atro-ph/0610390] have shown that the present theory a) admits of gravitational waves traveling with the speed of light in vacuum, and b) predicts a lensing effect additional to that of the classical GR. The extra term is proportional $\alpha$. 